\title{
Orbital-enriched flat-top partition of unity method for the Schrödinger eigenproblem
}

\author{
Clelia Albrecht ${ }^{\mathrm{a}}$, Constanze Klaar ${ }^{\mathrm{a}}$, John Ernest Pask ${ }^{\mathrm{b}}$, Marc Alexander Schweitzer ${ }^{\mathrm{a}, \mathrm{c}, *}$, \\ N. Sukumar ${ }^{\mathrm{d}, *}$, Albert Ziegenhagel ${ }^{\mathrm{a}}$ \\ ${ }^{a}$ Fraunhofer Institute for Algorithms and Scientific Computing SCAI, Schloss Birlinghoven, 53757 Sankt Augustin, \\ Germany \\ ${ }^{b}$ Physics Division, Lawrence Livermore National Laboratory, 7000 East Avenue, Livermore, CA 94550, U.S.A. \\ ${ }^{c}$ Institute for Numerical Simulation, Rheinische Friedrich-Wilhelms-Universität, Wegelerstr. 6, 53115 Bonn, Germany \\ ${ }^{d}$ Department of Civil and Environmental Engineering, University of California, Davis, CA 95616, U.S.A.
}

\begin{abstract}
Quantum mechanical calculations require the repeated solution of a Schrödinger equation for the wavefunctions of the system, from which materials properties follow. Recent work has shown the effectiveness of enriched finite element type Galerkin methods at significantly reducing the degrees of freedom required to obtain accurate solutions. However, time to solution has been adversely affected by the need to solve a generalized rather than standard eigenvalue problem and the ill-conditioning of associated systems matrices. In this work, we address both issues by proposing a stable and efficient orbital-enriched partition of unity method to solve the Schrödinger boundaryvalue problem in a parallelepiped unit cell subject to Bloch-periodic boundary conditions. In the proposed partition of unity method, the three-dimensional domain is covered by overlapping patches, with a compactly-supported weight function associated with each patch. A key ingredient in our approach is the use of non-negative weight functions that possess the flat-top property, i.e., each weight function is identically equal to unity over some finite subset of its support. This flattop property provides a pathway to devise a stable approximation over the whole domain. On each patch, we use $p$-th degree orthogonal (Legendre) polynomials that ensure $p$-th order completeness, and in addition include eigenfunctions of the radial Schrödinger equation. Furthermore, we adopt a variational lumping approach to construct a (block-)diagonal overlap matrix that yields a standard eigenvalue problem for which there exist efficient eigensolvers. The accuracy, stability, and efficiency of the proposed method is demonstrated for the Schrödinger equation with a harmonic potential as well as a localized Gaussian potential. We show that the proposed approach delivers optimal rates of convergence in the energy, and the use of orbital enrichment significantly reduces the number of degrees of freedom for a given desired accuracy in the energy eigenvalues while the stability of the enriched approach is fully maintained.
\end{abstract}

Keywords: quantum mechanics, partition of unity method, Bloch boundary conditions, variational mass lumping, enrichment functions, stability

\footnotetext{
${ }^{*}$ Corresponding authors

Email addresses: schweitzer@ins.uni-bonn.de (Marc Alexander Schweitzer), nsukumar@ucdavis.edu (N. Sukumar)
} 


\section{Introduction}

The Kohn-Sham (KS) equations of density functional theory (DFT) are the dominant theoretical formulation in quantum mechanical simulations of condensed matter (solids and liquids). The KS equations require the repeated solution of the steady-state Schrödinger and Poisson equations on a parallelepiped unit cell with Bloch-boundary conditions [1]. The solution of the Schrödinger equation is the most time-consuming part in KS-DFT calculations. The current state-of-the-art approach to solve the equations of KS-DFT is the planewave (PW) pseudopotential method that uses a Fourier basis set, and requires the solution of a discrete standard eigenproblem. It has been appreciated in recent years that enriched Galerkin methods [2-13] can be very competitive with PW methods in attaining the desired accuracy with comparable or far fewer degrees of freedom (basis functions). While early formulations [2, 6, 7, 10] employed direct enrichment, more recent approaches have employed discontinuous Galerkin [3, 4, 11] or partition of unity finite element formulations [2, 5, 9, 13] in order to strictly localize orbital enrichments, thus facilitating flexible approximation and efficient parallel implementation. In [5] it was shown that an enriched partition of unity finite element method (PUFEM) [14, 15] requires an order of magnitude fewer basis functions than current state-of-the-art PW based methods to attain the desired $1 \mathrm{mHa}$ /atom accuracy in total energy calculations. However, the ill-conditioning of the resulting system matrices and the need to solve a generalized rather than standard eigenvalue problem were key issues identified as adversely affecting time to solution in practice. In this work, we use a flat-top partition of unity method (PUM) [16-18] to address these issues in the approximation of the Schrödinger equation. Our flat-top PUM produces well-conditioned system (Hamiltonian and overlap) matrices and yields a standard eigenvalue problem via variational lumping. The approximation quality of our flat-top PUM is comparable to that reported in [2], but it overcomes the two main shortcomings of the PUFEM that arise in the solution of the Schrödinger eigenproblem. In addition to the electronic Schrödinger equation, the flat-top PUM with Bloch boundary conditions also holds promise in areas such as acoustic scattering [19], elastodynamics [20] and electromagnetics [21], where large-scale eigenproblems are solved and useful a priori information is available.

In condensed matter calculations, the Schrödinger equation is solved in a unit cell (parallelepiped domain $\Omega$ ) subject to Bloch-periodic boundary conditions (see Figure 1). In the flat-top partition of unity method, the domain $\Omega$ is covered by overlapping patches (see Figure 2 ) and each patch $i$ is associated with a weight function $\varphi_{i}(\boldsymbol{x})$ with support $\omega_{i}$ such that $\sum_{i} \varphi_{i}(\boldsymbol{x})=1$ and $\varphi_{i}(\boldsymbol{x}) \equiv 1$ on $\omega_{i}^{\mathrm{FT}} \subset \omega_{i}$ (see Figure 3 ). The local basis set $V_{i}$ on each patch consists of polynomials and/or non-polynomial (orbital enrichment) functions, and the global approximation is formed by linear combinations of the products of $\varphi_{i}(\boldsymbol{x})$ and functions from $V_{i}$. We perform local orthogonalization to ensure that all functions on a patch are linearly independent and thereby attain global stability [17], and adopt the variational lumping scheme [18] to realize a standard eigenproblem.

The remainder of the paper is organized as follows. In the next section, we state the strong and weak forms of the Schrödinger eigenproblem. In Section 3, we introduce the partition of unity method, where we present the proposed flat-top PUM in Section 3.1. The key steps in the local orthogonalization procedure to construct a stable global approximation are discussed in Section 3.2, and we describe the variational lumping scheme in Section 3.3. Numerical examples for the Schrödinger equation are presented in Section 4, where we show that the system matrices are well-conditioned and that the use of orbital-enrichment provides a very efficient solution visà-vis solely using polynomials over each patch. In addition, we also provide comparisons in the 
eigenspectrum when using the consistent overlap matrix versus the lumped overlap matrix, and the results reveal that the variational lumping scheme does not adversely affect the accuracy of the energy eigenvalues. We close with a few concluding remarks in Section 5 .

\section{The Schrödinger eigenproblem}

The stationary Schrödinger equation reads as

$$
\mathcal{H} \psi(x):=-\frac{1}{2} \nabla^{2} \psi(x)+V_{\mathrm{eff}}(\boldsymbol{x}) \psi(\boldsymbol{x})=\varepsilon \psi(\boldsymbol{x}) \quad \text { in } \Omega
$$

We consider a parallelepiped unit-cell $\Omega \subset \mathbb{R}^{3}$ with primitive lattice vectors $\boldsymbol{a}_{d}(d=1,2,3)$ to describe a periodic condensed matter system (see Figure 1). Thus, the effective potential and the charge density are periodic, i.e., they satisfy

$$
V_{\mathrm{eff}}(\boldsymbol{x}+\boldsymbol{R})=V_{\mathrm{eff}}(\boldsymbol{x}), \quad \rho(\boldsymbol{x}+\boldsymbol{R})=\rho(\boldsymbol{x}),
$$

whereas the solution of Schrödinger's equation $\psi$, the so-called wavefunction, satisfies Bloch's theorem

$$
\psi(\boldsymbol{x}+\boldsymbol{R})=\psi(\boldsymbol{x}) \exp (i \boldsymbol{k} \cdot \boldsymbol{R})
$$

for any lattice translation vector $\boldsymbol{R}=n_{1} \boldsymbol{a}_{1}+n_{2} \boldsymbol{a}_{2}+n_{3} \boldsymbol{a}_{3}$ with $n_{d} \in \mathbb{Z}(d=1,2,3)$ and wavevector $\boldsymbol{k}$. Note that for $\boldsymbol{k}=0$ ( $\Gamma$-point) the wavefunction is also periodic whereas for all other wavevectors there is a phase-shift $\exp (i \boldsymbol{k} \cdot \boldsymbol{R})$ associated with a translation by $\boldsymbol{R}$. In this situation the general problem (1) becomes

$$
\begin{aligned}
-\frac{1}{2} \nabla^{2} \psi(\boldsymbol{x})+V_{\mathrm{eff}}(\boldsymbol{x}) \psi(\boldsymbol{x}) & =\boldsymbol{\varepsilon} \psi(\boldsymbol{x}) & & \text { in } \Omega, \\
\psi\left(\boldsymbol{x}+\boldsymbol{a}_{d}\right) & =\exp \left(i \boldsymbol{k} \cdot \boldsymbol{a}_{d}\right) \psi(\boldsymbol{x}) & & \text { on } \Gamma_{d}, \\
\nabla \psi\left(\boldsymbol{x}+\boldsymbol{a}_{d}\right) \cdot \hat{\boldsymbol{n}}(\boldsymbol{x}) & =\exp \left(i \boldsymbol{k} \cdot \boldsymbol{a}_{d}\right) \nabla \psi(\boldsymbol{x}) \cdot \hat{\boldsymbol{n}}(\boldsymbol{x}) & & \text { on } \Gamma_{d},
\end{aligned}
$$

where $(\psi, \varepsilon)$ denotes an eigenpair consisting of the respective wavefunction $\psi$ and its associated energy $\varepsilon, \hat{\boldsymbol{n}}(\boldsymbol{x})$ is the outward unit normal at $\boldsymbol{x}$ and $\Gamma_{d}$ are the bounding faces of the domain $\Omega$, see Figure 1. Even though the boundary conditions and thus the wavefunction are complex-valued, the eigenvalues $\varepsilon \in \mathbb{R}$ due to the self-adjointness of the Hamiltonian $\mathcal{H}$.

In this paper, we are concerned with the numerical approximation of (4) by Galerkin methods and thus need to rewrite (4) in its respective weak form. To this end, we consider the function space

$$
\mathcal{V}:=\left\{v \in H^{1}(\Omega, \mathbb{C}): v\left(\boldsymbol{x}+\boldsymbol{a}_{d}\right)=v(\boldsymbol{x}) \exp \left(i \boldsymbol{k} \cdot \boldsymbol{a}_{d}\right) \text { on } \Gamma_{d}, d=1,2,3\right\}
$$

and test (4) with $v \in \mathcal{V}$ to attain

$$
a(v, \psi)=\varepsilon\langle v, \psi\rangle_{L^{2}(\Omega, \mathbb{C})} \text { for all } v \in \mathcal{V}
$$

with

$$
a(v, \psi):=\frac{1}{2} \int_{\Omega}\left(\overline{\nabla v(\boldsymbol{x})} \nabla \psi(\boldsymbol{x})+\overline{v(\boldsymbol{x})} V_{\mathrm{eff}}(\boldsymbol{x}) \psi(\boldsymbol{x})\right) d \boldsymbol{x}
$$

and

$$
\langle v, \psi\rangle_{L^{2}(\Omega, \mathbb{C})}:=\int_{\Omega} \overline{v(\boldsymbol{x})} \psi(\boldsymbol{x}) d \boldsymbol{x}
$$




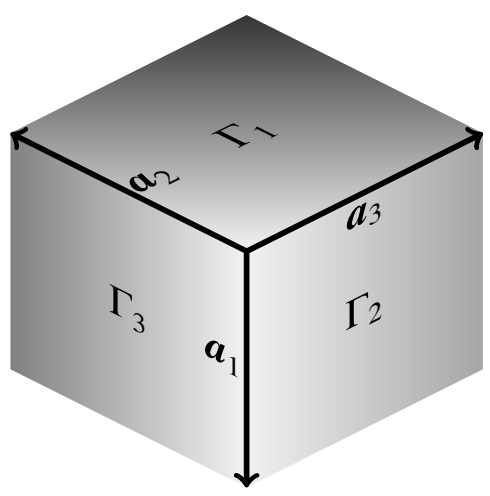

Figure 1: Sketch of a parallelepiped unit cell $\Omega$ spanned by primitive lattice vectors $\boldsymbol{a}_{d}$ with respective boundary segments $\Gamma_{d}(d=1,2,3)$.

after integration by parts [2]. Thus, choosing a finite-dimensional space $V_{M} \subset \mathcal{V}$ with basis functions $\phi_{i}$ to discretize (6) we obtain the generalized eigenproblem

$$
H \tilde{\psi}=\varepsilon S \tilde{\psi},
$$

where $H=\left(H_{i j}\right) \in \mathbb{C}^{M \times M}$ denotes the discrete Hamiltonian and $S=\left(S_{i j}\right) \in \mathbb{C}^{M \times M}$ is the so-called overlap (or consistent mass) matrix

$$
H_{i j}:=a\left(\phi_{j}, \phi_{i}\right) \text {, and } S_{i j}:=\left\langle\phi_{j}, \phi_{i}\right\rangle_{L^{2}(\Omega, \mathbb{C})} .
$$

The approximate eigenfunction $\psi \in V_{M}$ is given by

$$
\psi(\boldsymbol{x}):=\sum_{i=1}^{M} \psi_{i} \phi_{i}(\boldsymbol{x}),
$$

where $\tilde{\psi}=\left(\psi_{i}\right) \in \mathbb{C}^{M}$ denotes the associated coefficient vector. Throughout this paper we employ a particular partition of unity method [16, 22, 23] to construct the respective finite-dimensional space $V_{M}$ and its basis functions $\phi_{i}$.

\section{Partition of unity method}

The partition of unity method (PUM) is a generalization of the finite element method (FEM) that is typically employed for the spatial discretization of a partial differential equation (PDE), see for example [24, 25]. The notion of a PUM was coined in [14, 15] and is based on the special FEM developed in [26]. The abstract ingredients of a PUM are a partition of unity (PU) $\left\{\varphi_{i}: i=\right.$ $1, \ldots, N\}$ and a collection of local approximation spaces $V_{i}\left(\omega_{i}\right):=\operatorname{span}\left\langle\vartheta_{i}^{m}\right\rangle_{m=1}^{\operatorname{dim}\left(V_{i}\right)}$ defined on the patches $\omega_{i}:=\operatorname{supp}\left(\varphi_{i}\right)$ for $i=1, \ldots, N$ which overlap and whose union covers the computational domain $\Omega \subset \mathbb{R}^{d}$. With these two ingredients we define the PUM space

$$
V^{\mathrm{PU}}:=\sum_{i=1}^{N} \varphi_{i} V_{i}=\operatorname{span}\left\langle\varphi_{i} \vartheta_{i}^{m}\right\rangle ;
$$

that is, the basis functions of a PUM space are simply defined as the products of the PU functions $\varphi_{i}$ and the local approximation functions $\vartheta_{i}^{m}$. The PU functions provide the locality and global 

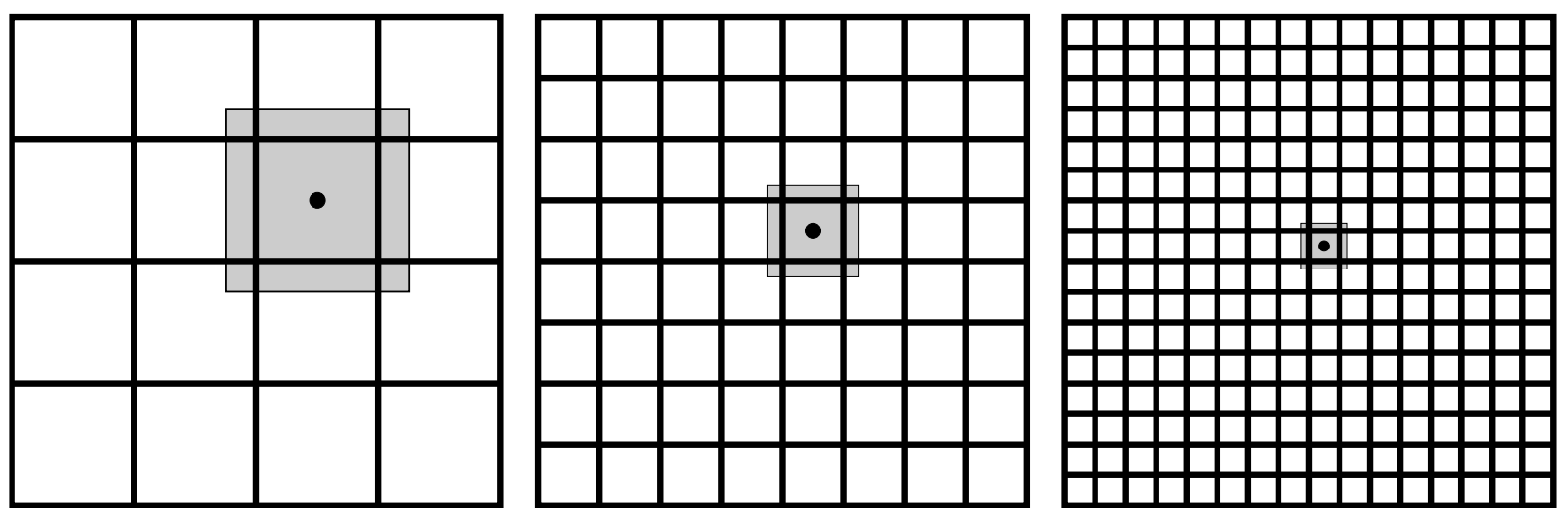

Figure 2: Schematic of a sequence of uniformly refined covers which come from the scaling of uniform grid cells in two dimensions. Depicted is a single cover patch $\omega_{i}=\prod_{l=1}^{d}\left(o_{i}^{l}-\alpha h, o_{i}^{l}+\alpha h\right)$ (gray) with $2 h=1 / 4,1 / 8,1 / 16($ left to right) and its center $o_{i}$.

regularity of the product functions whereas the functions $\vartheta_{i}^{m}$ equip $V^{\mathrm{PU}}$ with its approximation power. Note that the local approximation spaces $V_{i}$ can be chosen in a problem-dependent fashion and are independent of each other, i.e., any local basis $\vartheta_{i}^{m}$ may be employed on any patch $\omega_{i}$. In general the local approximation space $V_{i}:=\operatorname{span}\left\langle\vartheta_{i}^{n}\right\rangle$ associated with a particular patch $\omega_{i}$ of a PUM space $V^{\mathrm{PU}}$ consists of two parts: A smooth approximation space, for example polynomials $V_{i}^{\mathcal{P}}\left(\omega_{i}\right):=\operatorname{span}\left\langle\pi_{i}^{s}\right\rangle$, and a problem-dependent enrichment part $V_{i}^{\mathcal{E}}\left(\omega_{i}\right):=\operatorname{span}\left\langle\psi_{i}^{t}\right\rangle$, i.e.,

$$
\operatorname{span}\left\langle\vartheta_{i}^{n}\right\rangle=V_{i}\left(\omega_{i}\right)=V_{i}^{\mathcal{P}}\left(\omega_{i}\right)+V_{i}^{\mathcal{E}}\left(\omega_{i}\right)=\operatorname{span}\left\langle\pi_{i}^{s}, \psi_{i}^{t}\right\rangle .
$$

For the ease of notation, we make the following conventions. For an arbitrary function $u \in V^{\mathrm{PU}}$ with the representation

$$
u(x)=\sum_{i=1}^{N} \sum_{m=1}^{\operatorname{dim}\left(V_{i}\right)} u_{i}^{m} \varphi_{i}(x) \vartheta_{i}^{m}(x)=\sum_{i=1}^{N} \varphi_{i}(x) \sum_{m=1}^{\operatorname{dim}\left(V_{i}\right)} u_{i}^{m} \vartheta_{i}^{m}(x)=: \sum_{i=1}^{N} \varphi_{i}(x) u_{i}(x)
$$

we denote its associated overall coefficient vector by

$$
\tilde{u}=\left(u_{(i, m)}\right) \in \mathbb{R}^{\operatorname{dim}\left(V^{\mathrm{PU}}\right)} \text { with } \operatorname{dim}\left(V^{\mathrm{PU}}\right):=\sum_{i=1}^{N} \operatorname{dim}\left(V_{i}\right) .
$$

\subsection{Flat-top partition of unity}

The main difference between our PUM approach [22, 27] and most other generalized or extended FEM techniques, compare [24, 25], is that we employ a so-called flat-top PU instead of classical linear Lagrange FE functions, compare Figure 3. For the construction of such a flat-top PU let us first define a cover $C_{\Omega}:=\left\{\omega_{i}\right\}$ of the domain $\Omega$ with the help of a uniform regular mesh of mesh-width $2 h$ by an isotropic scaling of the mesh-cells

$$
C_{i}=\prod_{l=1}^{d}\left(o_{i}^{l}-h, o_{i}^{l}+h\right)
$$




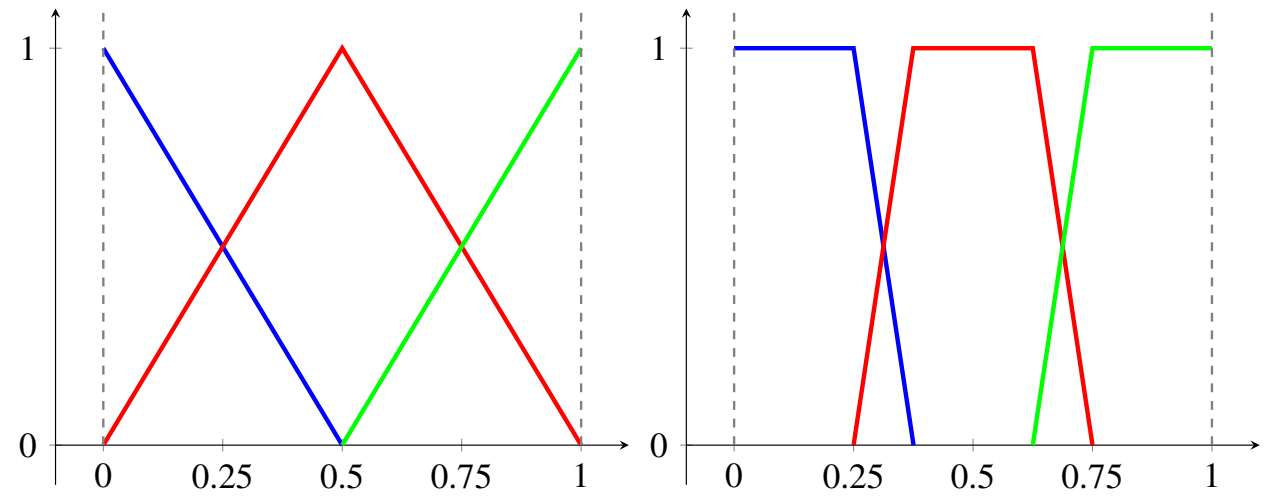

Figure 3: Partition of unity comprised of linear FE functions (left) and a flat-top PU (right) in one dimension.
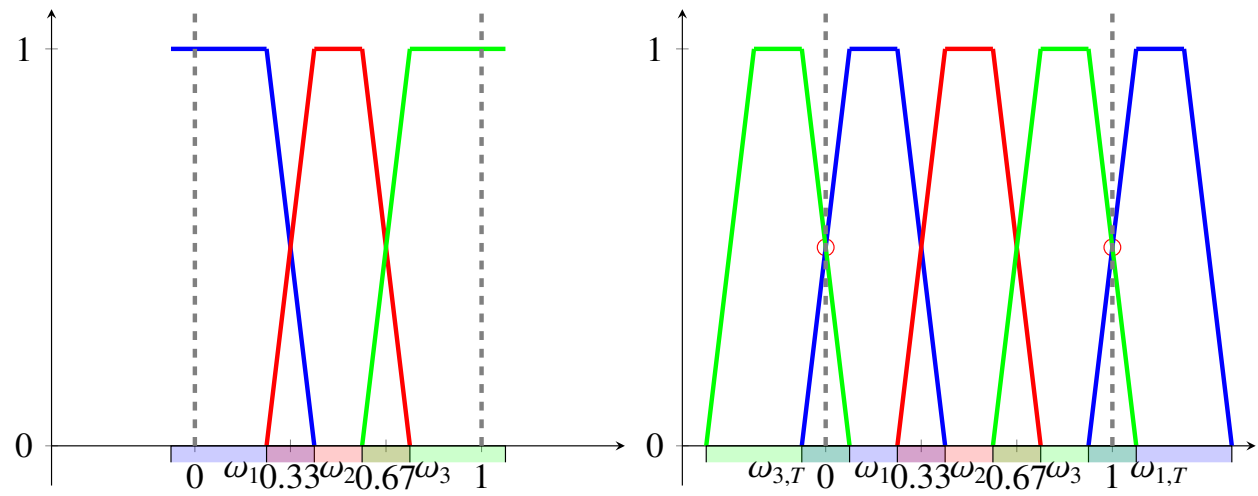

Figure 4: Comparison of one-dimensional flat-top PU weight functions for Dirichlet or Neumann boundary conditions (left) and periodic boundary conditions (right). To realize periodic weight functions we copy patches $\omega_{i}$ with $\omega_{i} \cap \partial \Omega \neq$ $\emptyset$ periodically (see also Figure 5 and [23]).
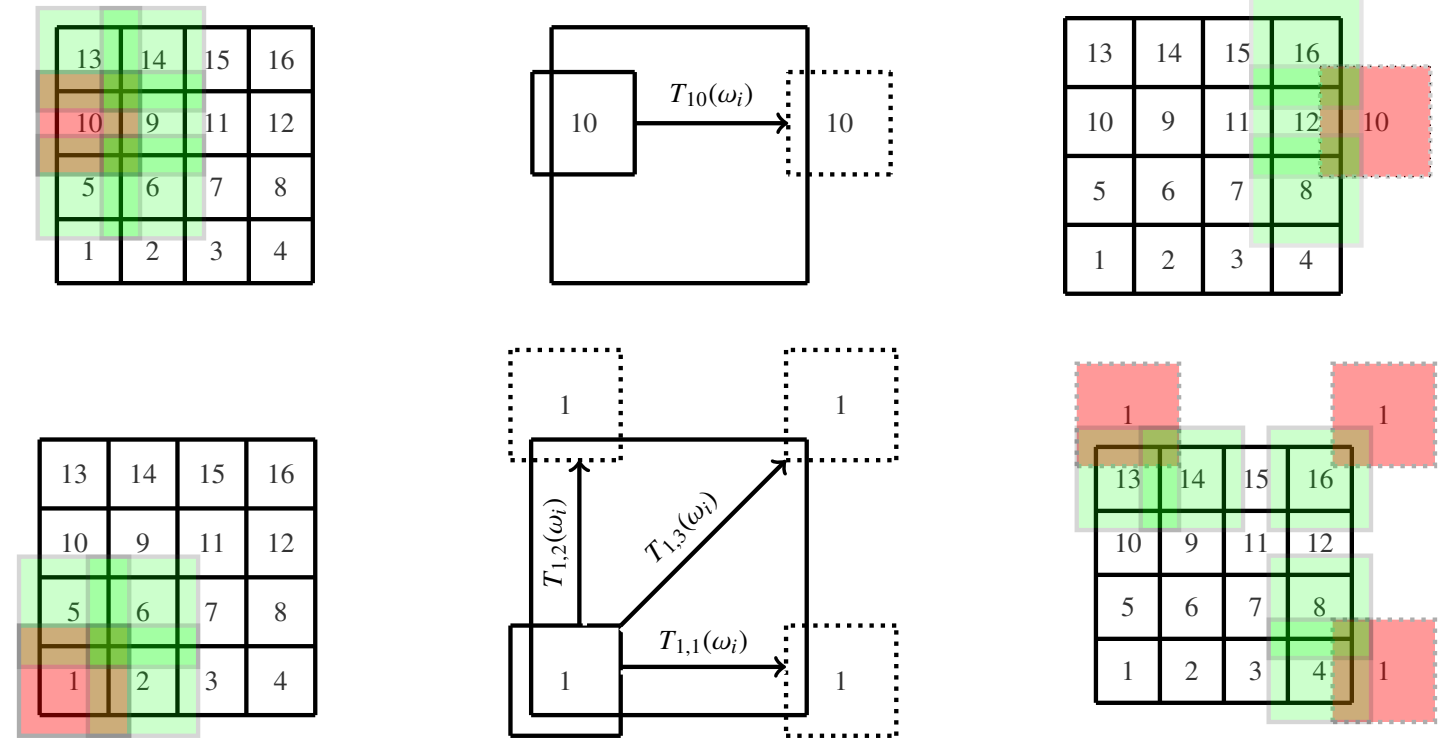

Figure 5: Schematic of the implementation of the periodicity of a PU function $\varphi_{i}(16)$ associated with a patch $\omega_{i}($ red) by generalization of the notion of neighboring or overlapping patches $\omega_{i} \cap \omega_{j} \neq \emptyset$ (green) at the boundary $\partial \Omega$ via periodic copies of patches $\omega_{i} \cap \partial \Omega \neq \emptyset$ (red), see [23] for details. 
i.e., we define the patches $\omega_{i}$ as

$$
\omega_{i}:=\prod_{l=1}^{d}\left(o_{i}^{l}-\alpha h, o_{i}^{l}+\alpha h\right), \quad \text { with } \alpha>1,
$$

see Figure 2, To obtain a PU on a cover $C_{\Omega}$ with $N:=\operatorname{card}\left(C_{\Omega}\right)$ we define a weight function $W_{i}: \Omega \rightarrow \mathbb{R}$ with $\operatorname{supp}\left(W_{i}\right)=\omega_{i}$ for each cover patch $\omega_{i}$ by

$$
W_{i}(x)= \begin{cases}W \circ T_{i}(x), & x \in \omega_{i} \\ 0, & \text { otherwise }\end{cases}
$$

with the affine transforms $T_{i}: \bar{\omega}_{i} \rightarrow[-1,1]^{d}$ and $\mathcal{W}:[-1,1]^{d} \rightarrow \mathbb{R}$ any non-negative compactly supported function, such as a B-spline. By normalizing these weight functions we obtain the functions

$$
\varphi_{i}(x):=\frac{W_{i}(x)}{\sum_{l \in C_{i}} W_{l}(x)},
$$

where we define the local neighborhood $C_{i}:=\left\{l: \omega_{l} \cap \omega_{i} \neq \emptyset\right\}$ of a patch $\omega_{i}$ in a slightly more general way to account for the required periodicity of the basis functions, see Figures 4 and 5 To impose Bloch-periodic boundary conditions, we multiply the entries of the resulting system matrices in matrix blocks that correspond to Bloch-boundary patches with the appropriate Bloch phase factor. This results in a periodic PU and Bloch-periodic basis, analogous to the procedure in [2]. Note that the PU (16) is non-negative since the employed weight functions are non-negative and that the $\varphi_{i}$ satisfy the flat-top property for any $\alpha \in(1,2)$, see [28, 29]. Due to this construction we can easily control the overlap of the patches and thereby the size of the flat-top region by the parameter $\alpha \in(1,2)$. Note that for $\alpha=1$ our PUM degenerates to a discontinuous Galerkin approach whereas with the choice of $\alpha=2$ and linear B-spline weights in (15) our PUM yields the PUFEM.

\subsection{Orbital enrichment and stability}

The approximation power of an enriched PUM is mostly obtained by the choice of high quality enrichment space $V^{\mathcal{E}}$ (enrichment functions $\psi_{i}^{t}$ ) on the patches $\omega_{i}$ for the problem at hand. For instance, the use of generalized harmonic polynomials or local spaces based on planewaves have been employed successfully for smooth problems in [14, 30, 31], whereas for crack propagation problems the use of discontinuous and singular enrichment functions is appropriate, see e.g. [25, 32, 33]. In the context of the Schrödinger and Poisson equations of Kohn-Sham density functional theory, enrichment functions $\psi_{i}^{t}$ constructed from isolated-atom solutions were employed in [5, 9. 10], as in previous work [2] in the context of model densities and potentials. Our general flat-top PUM allows for the use of arbitrary problem-dependent enrichment functions and thus we anticipate that our PUM has essentially the same convergence properties as for example the PUFEM of [2, 5, 10] when we employ the same enrichment functions.

The fundamental difference of our approach from the PUFEM of [2, 5, 10] is that we employ a flat-top PU [17, 27, 34] to overcome the two major challenges encountered in the PUFEM: illconditioning of the overlap matrix and the need for the solution of a generalized eigenproblem.

Let us first consider the ill-conditioning of the overlap matrix, i.e., the $L^{2}$-stability of (12), which can be encountered in all enriched approximations [24, 25, 35-37]. For the smooth space $V_{i}^{\mathcal{P}}$ 
in (13) we employ a local basis $\pi_{i}^{s}$ on $\omega_{i}$, i.e., $\pi_{i}^{s}=p_{s} \circ T_{i}$ and $\left\{p_{s}\right\}$ denotes a stable basis on $[-1,1]^{d}$, for instance Legendre-polynomials. The enrichment functions $\psi_{i}^{t}$ however are often given as global functions $\eta^{t}$ on the computational domain $\Omega$ since they are designed to capture special behavior of the global solution at a particular location. Therefore, the restrictions $\psi_{i}^{t}:=\left.\eta^{t}\right|_{\omega_{i}}$ of the enrichment functions $\eta^{t}$ to a particular patch $\omega_{i}$ may be ill-conditioned or even linearly dependent on $\omega_{i}$, even if the enrichment functions $\eta^{t}$ are well-conditioned on the global domain. Furthermore, the coupling between the spaces $V_{i}^{\mathcal{P}}$ and $V_{i}^{\mathcal{E}}$ on the patch $\omega_{i}$ must be considered. The set of functions $\left\{\pi_{i}^{s}, \psi_{i}^{t}\right\}$ will degenerate from being a basis of $V_{i}$ to yielding a generating system if the restricted enrichment functions $\psi_{i}^{t}=\left.\eta^{t}\right|_{\omega_{i}}$ can be well-approximated by polynomials $\pi_{i}^{s}$ on the patch $\omega_{i}$. Moreover, in the general case when (12) employs an arbitrary non-flat-top PU, e.g., a PU built from FE, we also need to consider the interactions of the overlapping local approximation spaces $V_{i}$ and $V_{j}(13)$ on neighboring patches $\omega_{i} \cap \omega_{j} \neq \emptyset$ which essentially introduces global constraints and therefore renders the use of an arbitrary non-flat-top PU computationally infeasible. If we, however, restrict ourselves to the use of a flat-top PU, all issues that may result in ill-conditioning can be efficiently resolved solely on the local patches $\omega_{i}$, see [17] for further details.

To attain a stable basis of $V^{\mathrm{PU}}$ for arbitrary local approximation spaces $V_{i}$ with a flat-top PU $\left\{\varphi_{i}\right\}$, we essentially need to be able to identify a local enrichment space $V_{i}^{\mathcal{D}}$ such that we can rewrite (13) as

$$
V_{i}=V_{i}^{\mathcal{P}}+V_{i}^{\mathcal{E}}=V_{i}^{\mathcal{P}} \oplus V_{i}^{\mathcal{D}}
$$

To this end, we choose an inner product on the patch $\omega_{i}$ and construct $V_{i}^{\mathcal{D}}$ such that $V_{i}^{\mathcal{P}} \perp V_{i}^{\mathcal{D}}$ holds. The respective stability transformation

$$
P: V^{\mathrm{PU}}=\sum_{i=1}^{N} \varphi_{i}\left(V_{i}^{\mathcal{P}}+V_{i}^{\mathcal{E}}\right) \rightarrow \sum_{i=1}^{N} \varphi_{i}\left(V_{i}^{\mathcal{P}} \oplus V_{i}^{\mathcal{D}}\right)
$$

can in fact be computed efficiently on-the-fly by partial orthogonalization with respect to the chosen inner product and only involves local operations on the patches [17]. The particular stability transformation on a patch $\omega_{i}$ depends on the choice of the inner product and works in four steps starting from a local matrix

$$
M_{i}=\left(\begin{array}{cc}
M_{i ; \mathcal{P}, \mathcal{P}} & M_{i ; \mathcal{P}, \mathcal{E}} \\
M_{i ; \mathcal{E}, \mathcal{P}} & M_{i ; \mathcal{E}, \mathcal{E}}
\end{array}\right)
$$

arising from Gram matrix of all basis functions $\pi_{i}^{s}$ of $V_{i}^{\mathcal{P}}$ and $\psi_{i}^{t}$ of $V_{i}^{\mathcal{E}}$ in the chosen inner product. First, all basis functions of $V_{i}^{\mathcal{P}}$ and $V_{i}^{\mathcal{E}}$ are scaled appropriately with respect to the employed inner product, so that the induced norm of all functions is identical. Then, we compute eigenvalue decompositions of $M_{i ; \mathcal{P}, \mathcal{P}}$ and $M_{i ; \mathcal{E}, \mathcal{E}}$ individually and use thresholding of small eigenvalues to eliminate any instabilities within $V_{i}^{\mathcal{P}}$ and $V_{i}^{\mathcal{E}}$, so that we can transform $M_{i}$ to attain the form

$$
M_{i}^{(1)}=\left(\begin{array}{cc}
\mathbb{I}_{\mathcal{P}} & M_{i ; \mathcal{P}, \mathcal{E}}^{(1)} \\
M_{i ; \mathcal{E}, \mathcal{P}}^{(1)} & \mathbb{I}_{\mathcal{E}}
\end{array}\right) .
$$

Then, the overlap of the two spaces $V_{i}^{\mathcal{P}}$ and $V_{i}^{\mathcal{E}}$ is removed by partial orthogonalization (Schur complement) which yields

$$
M_{i}^{(2)}=\left(\begin{array}{cc}
\mathbb{I}_{\mathcal{P}} & 0 \\
0 & M_{i ; \mathcal{D}, \mathcal{D}}^{(2)}
\end{array}\right)
$$


Thus, with the help of an eigenvalue decomposition of $M_{i ; \mathcal{D}, \mathcal{D}}^{(2)}$ we obtain an orthonormal basis of $V_{i}$ with respect to the employed inner product. And so the problem of ill-conditioning which is encountered in PUFEM can be resolved in a flat-top PUM while fully maintaining the improved approximation quality due to enrichment.

\subsection{Variational mass lumping}

The second drawback of the PUFEM identified in [5] is related to the fact that the Galerkin discretization of (4) yields a Hermitian generalized eigenproblem (9) where $H$ and $S$ are in general sparse but not diagonal matrices, which renders the solution of 97 more challenging since the computation of $S^{-1}$ is implicitly required, i.e., one effectively needs to solve the standard eigenproblem

$$
S^{-1} H \tilde{u}=\varepsilon \tilde{u} .
$$

In the context of classical (linear) FEM one therefore often replaces the overlap (or consistent mass) matrix $S$ by an approximation $\bar{S}$ whose inverse $\bar{S}^{-1}$ can be computed much more efficiently, e.g., if $\bar{S}$ is diagonal, by so-called mass-lumping [38-40]. Various constructions for $\bar{S}$ exist in the FEM context [41], yet these approaches are in general not directly applicable to a PUM or any enriched approximation scheme due to the non-polynomial and non-interpolatory character of the employed basis functions.

Fortunately, there is a natural and rather simple approach to the construction of an appropriate approximation of the overlap matrix for the PUM in general [18]. It is applicable to any nonnegative PU $\left\{\varphi_{i}\right\}$ and arbitrary local approximation spaces (13) (e.g., higher order polynomials, discontinuous, and singular enrichments) and utilizes only the special product structure of the PUM basis functions $\varphi_{i} \vartheta_{i}^{n}$.

To introduce this variational lumping scheme for our PUM, recall that the consistent overlap matrix is given by

$$
S=\left(S_{(i, n),(j, m)}\right), \quad S_{(i, n),(j, m)}=\left\langle\varphi_{j} \vartheta_{j}^{m}, \varphi_{i} \vartheta_{i}^{n}\right\rangle_{L^{2}(\Omega, \mathbb{C})},
$$

where our flat-top PU functions $\varphi_{i}$ and $\varphi_{j}$ are non-negative. Thus, the entries of the overlap matrix are given by the inner products in $L^{2}(\Omega, \mathbb{C})$ of our PUM basis functions. In [18] it was shown that the global $L^{2}(\Omega, \mathbb{C})$ inner product in $(18)$ can be replaced by local weighted $L^{2}\left(\omega_{i}, \mathbb{C} ; \varphi_{i}\right)$ inner products without diminishing the global convergence behavior, since there holds the equivalence

$$
\left\langle f, \varphi_{i} \vartheta_{i}^{n}\right\rangle_{L^{2}(\Omega, \mathbb{C})}=\int_{\Omega} f \varphi_{i} \vartheta_{i}^{n} d x=\int_{\Omega \cap \omega_{i}} f \varphi_{i} \vartheta_{i}^{n} d x=:\left\langle f, \vartheta_{i}^{n}\right\rangle_{L^{2}\left(\Omega \cap \omega_{i}, \mathbb{C} ; \varphi_{i}\right)}
$$

for an arbitrary function $f \in L^{2}(\Omega, \mathbb{C})$. With the help of these local inner products, we therefore obtain an approximate overlap matrix

$$
\bar{S}=\left(\bar{S}_{(i, n),(j, m)}\right), \quad \bar{S}_{(i, n),(j, m)}=\left\{\begin{array}{cc}
0 & i \neq j \\
\left\langle\vartheta_{i}^{m}, \vartheta_{i}^{n}\right\rangle_{L^{2}\left(\Omega \cap \omega_{i}, \mathbb{C} ; \varphi_{i}\right)} & i=j
\end{array}\right.
$$

which is block-diagonal and symmetric positive definite for any choice of the local approximation spaces $V_{i}$, see [18] for further details. Therefore, an approximate solution of (9) can be obtained very efficiently via

$$
H \tilde{u}=\varepsilon \bar{S} \tilde{u} .
$$

In fact, choosing the local weighted $L^{2}\left(\omega_{i}, \mathbb{C} ; \varphi_{i}\right)$ inner products also in the stability transformation (17) yields $\bar{S}=\mathbb{I}$, so that (20) becomes $H \tilde{u}=\varepsilon \tilde{u}$ and the expensive solution of the generalized eigenproblem encountered in the PUFEM can be completely circumvented in our flat-top PUM. 


\section{Numerical results}

In this section we present some numerical results obtained with our flat-top PUM. We consider two benchmark problems defined on three-dimensional unit cells [2] to validate our implementation and assess the accuracy and efficiency in terms of the degrees of freedom (DOFs) of the flat-top PUM with respect to the Schrödinger eigenproblem with periodic and Bloch-periodic boundary conditions. The two main objects of interest of our study, however, are the conditioning of the consistent and lumped overlap matrices (before and after the stabilization (17)) and the effect our lumping scheme (19) has on the quality of the approximation. Since absolute runtime is not the focus of this study, we employed a simple tensor-product $6 \times 6 \times 6$ Gauß integration rule on subdivided cover cells [16, 22].

\subsection{Three-dimensional quantum harmonic oscillator}

As in [2], the first benchmark problem we consider is the Schrödinger equation (4) with harmonic potential

$$
V(|x-\tau|)=\frac{|x-\tau|^{2}}{2}
$$

under periodic boundary conditions $(\boldsymbol{k}=0)$. Note that this potential itself is not periodic. As our unit cell $\Omega$, we take a cuboid cell with primitive lattice vectors $\boldsymbol{a}_{1}:=a(1,0,0), \boldsymbol{a}_{2}:=a(0,1.1,0)$ and $\boldsymbol{a}_{3}:=a(0,0,1.2)$. As lattice parameter we choose $a=5$ au and the potential center is given by $\tau:=\frac{a_{1}+a_{2}+a_{3}}{2}$.

On each patch, the polynomial approximation space $V_{i}^{\mathcal{P}}$ contains Legendre polynomials up to total degree $p=1,2,3$, while the enrichment space $V_{i}^{\mathcal{E}}$ consists of periodic lattice sums of the infinite box eigenfunctions $\psi_{n l m}^{\prime}(\boldsymbol{x})=R_{n l}(r) Y_{l m}(\theta, \phi)$,

$$
\psi_{n l m}=\sum_{\boldsymbol{R}} \psi_{n l m}^{\prime}(|\boldsymbol{x}-\boldsymbol{\tau}-\boldsymbol{R}|)
$$

over lattice translation vectors $\boldsymbol{R}=i_{1} \boldsymbol{a}_{1}+i_{2} \boldsymbol{a}_{2}+i_{3} \boldsymbol{a}_{3}$, to enrich each of the ten lowest states. Here, $Y_{l m}$ are the analytically given spherical harmonics and $R_{n l}$ is the radial part of the eigenfunction, which is obtained by solving the radial Schrödinger equation [2]. Thus, the radial components are given numerically, i.e., at discrete points. To ensure a compact support of $R_{n l}$ we multiply its discrete values by a $C^{3}$ cut-off function [2]

$$
h\left(r, r_{0}\right):= \begin{cases}1+\frac{20 r^{7}}{r_{0}^{7}}-\frac{70 r^{6}}{r_{0}^{6}}+\frac{84 r^{5}}{r_{0}^{5}}-\frac{35 r^{4}}{r_{0}^{4}}, & r \leq r_{0} \\ 0, & r>r_{0}\end{cases}
$$

where we choose $r_{0}=6 \mathrm{au}$. The resulting product is then interpolated by a quintic spline.

For our numerical experiments, we measure the absolute error of the computed eigenvalues with respect to a cubic finite element reference solution, computed on a $64 \times 64 \times 64$ mesh which is accurate to 7 digits (and yields $\lambda_{1}^{\text {ref }}=1.4917524 \mathrm{Ha}$ and $\sum_{i=1}^{10} \lambda_{i}^{\text {ref }}=29.7715084 \mathrm{Ha}$ ).

First, we assess the accuracy of the consistent method. To this end, we first apply our flat-top PUM with linear, quadratic and cubic polynomial spaces on uniformly refined covers without any orbital enrichment. As stretch factor (14) we choose $\alpha=1.1$. Here, we anticipate that the flat-top PUM converges with rates that are comparable to classical finite element methods of the respective 


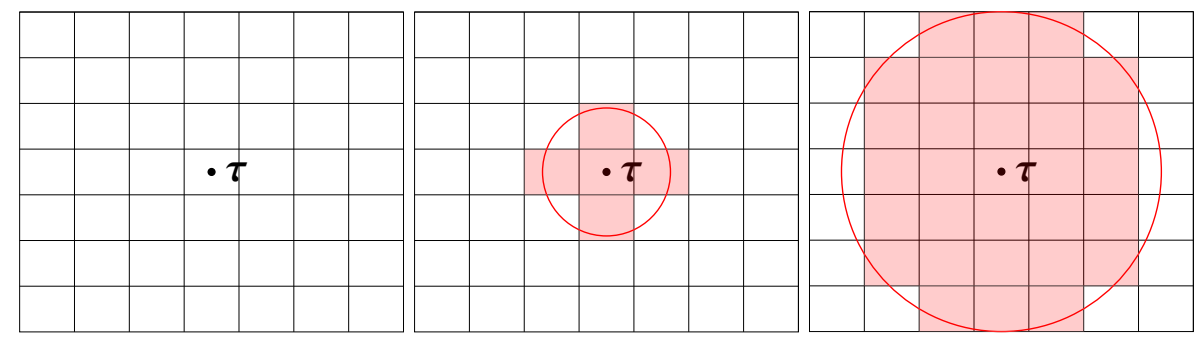

Figure 6: Sketch of refinement by increasing the enrichment radius $r_{e}$ in two dimensions. We only enrich a patch if its center point lies within the ball of radius $r_{e}$ centered at the potential center $\tau$. Here, this ball and enriched patches are colored in red. Depicted are three different values for $r_{e}: 0.0$ au (left), 1.0 au (center) and 2.5 au (right) on a $7 \times 7$ cover.

order. Then, we use a fixed uniform cover and use enriched local approximation spaces, where we increase the enrichment support radius from 0 to 4 au (see Figure 6). In the first case we do not use any enrichments at all, in the latter we enrich each patch whose center lies within the support radius of the enrichment center $\tau$. The results are shown in Figure 7 . From the depicted plots we can see that the convergence behavior of the non-enriched method is just as expected, while it is also evident that using enrichment functions is very beneficial in terms of accuracy: significantly less than 500 DOFs (e.g., 320 DOFs with linear polynomials on a $4 \times 4 \times 4$ cover and 297 DOFs with quadratic polynomials on a $3 \times 3 \times 3$ cover) yield an error of less than $10^{-3} \mathrm{Ha}$, which is a typical requirement in quantum mechanical calculations. Apart from reducing DOFs, enrichment also reduces the overall wall clock time of the method: in our experiments, the computation (including setup, assembling of matrices and solving the EVP) on a $3 \times 3 \times 3$ cover with quadratic polynomials, a single enrichment function for the lowest eigenvalue and an enrichment support radius of 4 au took 7.03 seconds on 8 cores, while the non-enriched computation with quadratic polynomials on a $16 \times 16 \times 16$ cover took 192.67 seconds on 8 cores. Both methods yield about the same absolute error for the lowest eigenvalue $\lambda_{1}$ (see Figure 7), in both cases less than $10^{-3}$ Ha. Note that there is a distinct difference in the PUM functions $\varphi_{i} \vartheta_{i}^{m}$ on even (e.g., $\left.4 \times 4 \times 4\right)$ and odd (e.g., $3 \times 3 \times 3$ ) covers. In the odd case, the potential center is located within the flat-top region of a single patch whereas for the even case $\tau$ is contained in the overlap of eight neighboring patches. Thus, the results summarized in Figure 7 show that our approach is also fully robust with respect to the relative position of $\tau$.

To properly evaluate the benefits of the lumped method, we first conduct the same experiment as above, only this time using a lumped overlap matrix and summarize the results in Figure 9 . We observe that the convergence behavior is practically identical to that of the consistent method, however, with a slightly larger absolute error. Apart from being almost as accurate as the consistent method, using the lumped overlap matrix has its own benefits: First of all we can reduce the generalized eigenproblem (9) to a standard eigenproblem as described in Section 3.3, which saves a lot of computational time, especially for larger problems (see Figure 8). In the context of self-consistent Kohn-Sham calculations this is especially important, as it enables the use of highly efficient algorithms to refine eigenvectors in each self-consistent-field iteration [11, 42]. Computational time for the overall method is also again saved by using enrichment functions: while the computation on a $16 \times 16 \times 16$ cover using quadratic polynomials without enrichments took 151.78 seconds on 8 cores, almost the same absolute error for the lowest eigenvalue $\lambda_{1}$ was achieved by 

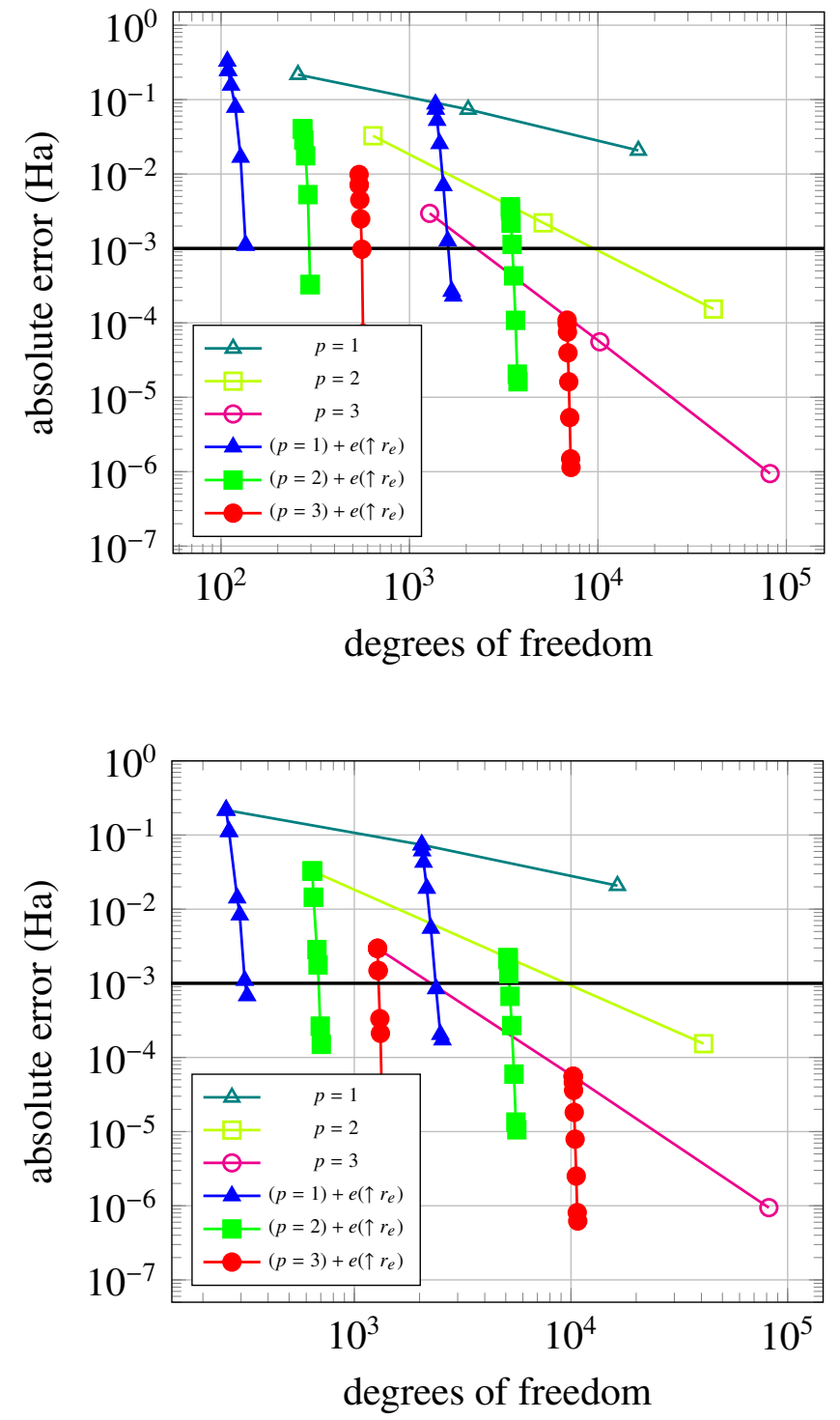

Figure 7: Convergence history of the lowest eigenvalue $\lambda_{1}$ for the harmonic oscillator potential 21] attained for different refinement schemes. We consider a purely polynomial approximation $(p=1,2,3)$ on a sequence of uniformly refined covers, and a refinement by increasing the enrichment radius (compare Figure 6) with a single enrichment function on a fixed uniform cover (top: $3 \times 3 \times 3,7 \times 7 \times 7$; bottom: $4 \times 4 \times 4,8 \times 8 \times 8$ ) that is labeled by $p=1,2,3+e \uparrow r_{e}$. All results were obtained with the consistent overlap matrix. 

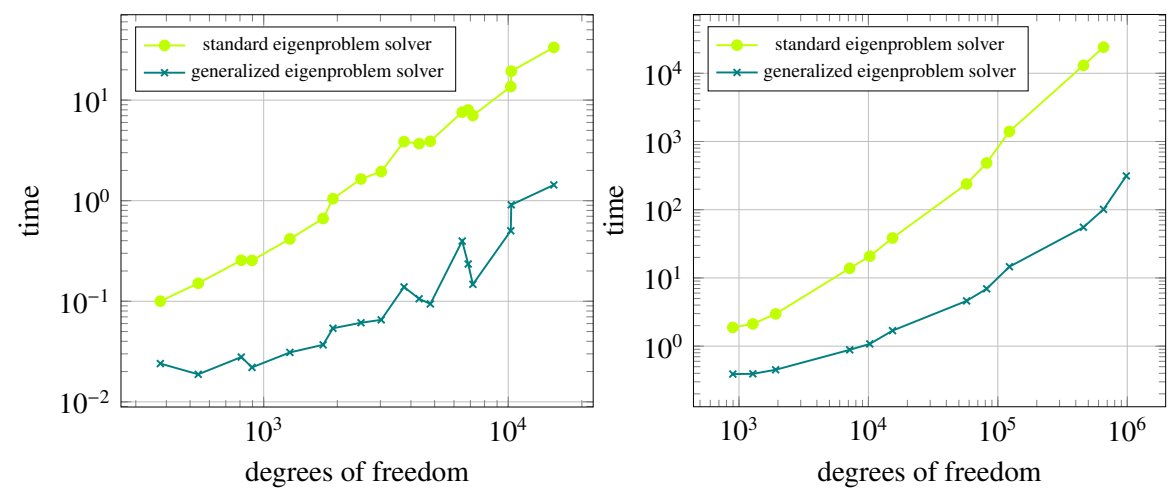

Figure 8: Wall clock time measured for the solution of the generalized and standard eigenproblems for the harmonic oscillator potential (21), using the default SLEPc eigenvalue solver, polynomials of degree $p=1,2,3$ and ten enrichments on an $n \times n \times n$ cover. All these data points are in one plot, sorted by number of DOFs (left: $n=3,4,5,6,7,8$ on 16 cores; right: $n=4,8,16,32$ on 64 cores).

Table 1: Measured condition numbers with and without stabilization (17) with respect to the local weighted $L^{2}$ inner product $L^{2}\left(\omega_{i}, \mathbb{C} ; \varphi_{i}\right)$ for the consistent and the lumped overlap matrix obtained with $p=3$ on a $7 \times 7 \times 7$ uniform cover for increasing enrichment radius $r_{e}$ and ten enrichment functions per enriched patch.

\begin{tabular}{cccccc}
\hline \multicolumn{3}{c}{ consistent overlap matrix } & \multicolumn{2}{c}{ lumped overlap matrix } \\
\hline$r_{e}$ & DoFs & without $[17$ & with $[17)$ & without $[17]$ & with $[17]$ \\
\hline 0.0 & 6,860 & $4 \cdot 10^{3}$ & $7 \cdot 10^{2}$ & $6 \cdot 10^{1}$ & $1 \cdot 10^{0}$ \\
0.5 & 6,870 & $2 \cdot 10^{11}$ & $9 \cdot 10^{2}$ & $8 \cdot 10^{10}$ & $1 \cdot 10^{0}$ \\
1.0 & 6,930 & $4 \cdot 10^{11}$ & $2 \cdot 10^{3}$ & $9 \cdot 10^{10}$ & $1 \cdot 10^{0}$ \\
1.5 & 7,150 & $5 \cdot 10^{11}$ & $3 \cdot 10^{3}$ & $9 \cdot 10^{10}$ & $1 \cdot 10^{0}$ \\
2.0 & 7,590 & $6 \cdot 10^{11}$ & $5 \cdot 10^{3}$ & $1 \cdot 10^{11}$ & $1 \cdot 10^{0}$ \\
2.5 & 8,270 & $1 \cdot 10^{12}$ & $4 \cdot 10^{3}$ & $2 \cdot 10^{11}$ & $1 \cdot 10^{0}$ \\
3.0 & 9,090 & $2 \cdot 10^{12}$ & $5 \cdot 10^{3}$ & $4 \cdot 10^{11}$ & $1 \cdot 10^{0}$ \\
3.5 & 9,890 & $6 \cdot 10^{13}$ & $5 \cdot 10^{3}$ & $7 \cdot 10^{12}$ & $1 \cdot 10^{0}$ \\
4.0 & 10,210 & $3 \cdot 10^{14}$ & $5 \cdot 10^{3}$ & $2 \cdot 10^{13}$ & $1 \cdot 10^{0}$ \\
\hline
\end{tabular}

using a $3 \times 3 \times 3$ cover with quadratic polynomials, a single enrichment function for the lowest eigenvalue and an enrichment support radius of 4 au in 5.87 seconds on 8 cores.

Furthermore, we can overcome the problem of large condition numbers that arises in the PUFEM [5] using the stable transformation introduced in Section 3.2. The measured condition numbers for both consistent and lumped overlap matrices are displayed before and after applying the stable transformation (17) in Table 1. Here we see that for both types of overlap matrices, the condition number deteriorates quickly with increasing enrichment support radius without our stabilization (17). The same observation was made in [5] for the PUFEM. However, after applying the stable transformation, we can dramatically reduce the condition number to the range of classical FE overlap matrices for the consistent case. Combining the stabilization (17) with our lumping approach (19) yields the optimal behavior $\bar{S}=\mathbb{I}$ so that (20) becomes a well-conditioned standard eigenproblem. Thus, our flat-top PUM overcomes the two main drawbacks of the PUFEM and enables the stable use of enrichment functions on every patch and arbitrary potentials so that high-fidelity approximations can be attained with extremely small numbers of DOFs in a robust fashion. For example, the PUFEM employed in [2] yields an accuracy of $10^{-3} \mathrm{Ha}$ for this example with 948 DOFs using enriched cubic FE where the condition number of the overlap matrix is of the 

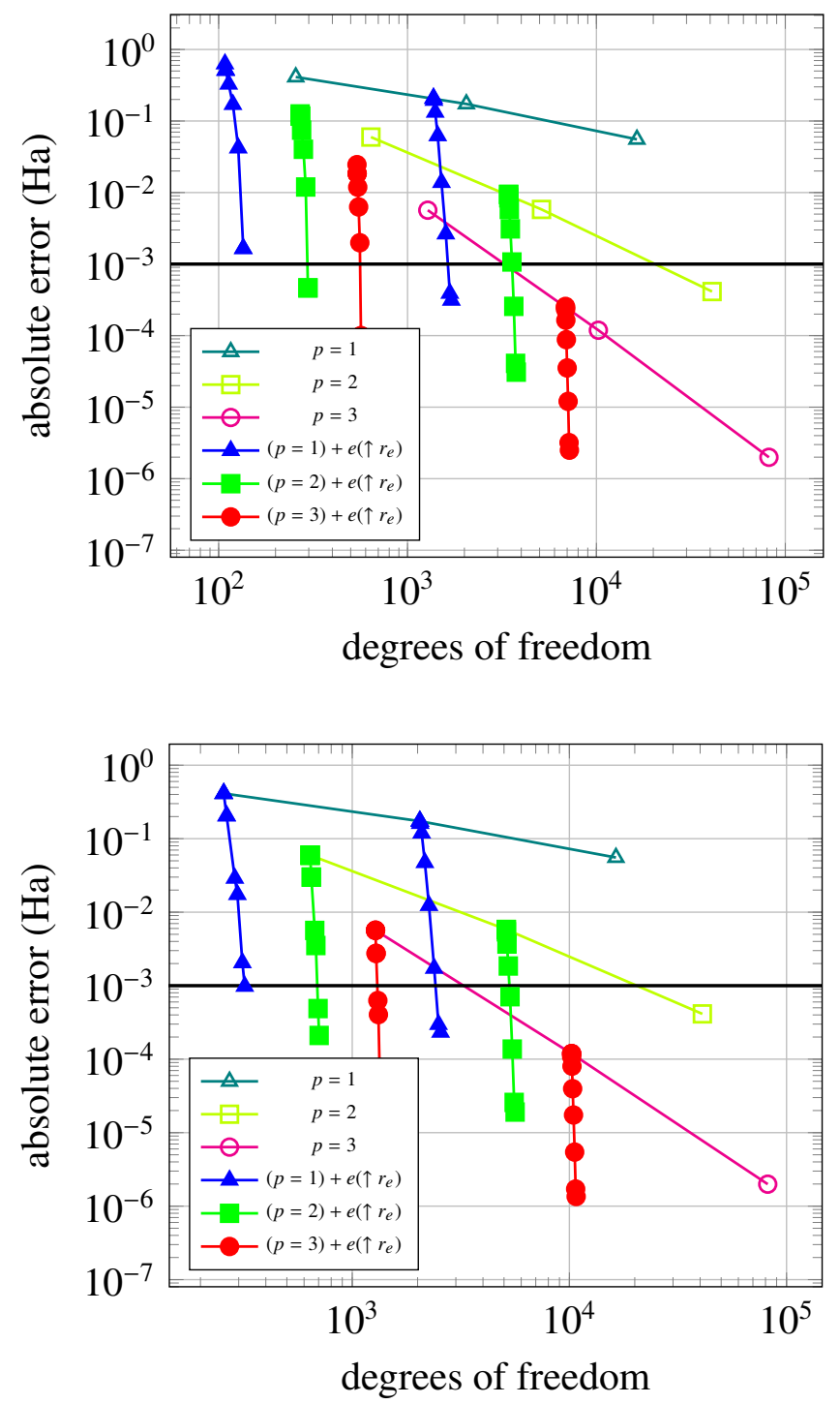

Figure 9: Convergence history of the lowest eigenvalue $\lambda_{1}$ for the harmonic oscillator potential (21) attained for different refinement schemes. We consider a purely polynomial approximation $(p=1,2,3)$ on a sequence of uniformly refined covers, and a refinement by increasing the enrichment radius (compare Figure 6) with a single enrichment function on a fixed uniform cover (top: $3 \times 3 \times 3,7 \times 7 \times 7$; bottom: $4 \times 4 \times 4,8 \times 8 \times 8$ ) that is labeled by $p=1,2,3+e \uparrow r_{e}$. All results were obtained with the lumped overlap matrix. 


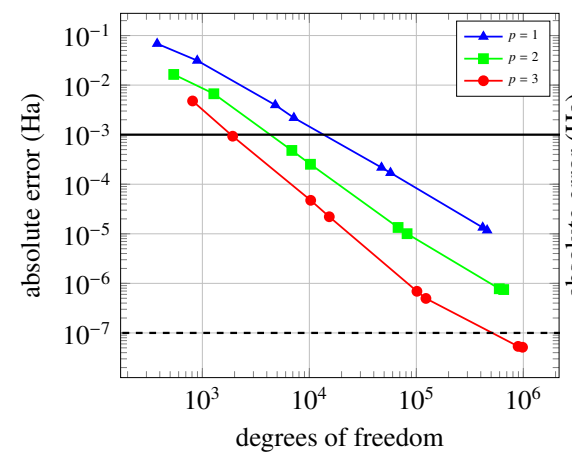

(a) $\alpha=1.1$

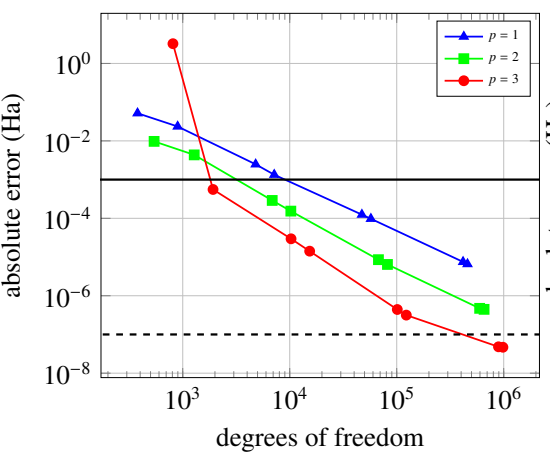

(b) $\alpha=1.2$

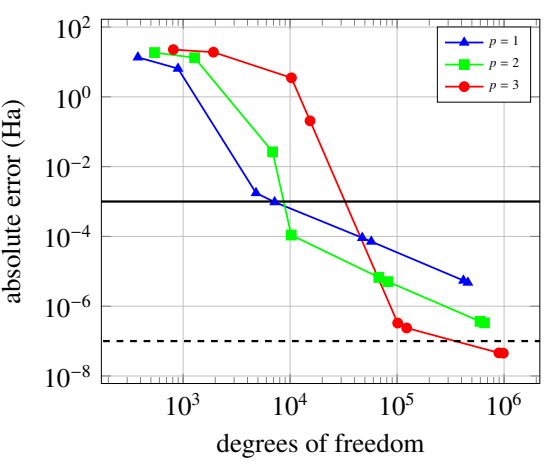

(c) $\alpha=1.3$

Figure 10: Convergence history of the sum of the ten lowest eigenvalues for the harmonic oscillator potential 21] attained for different values of $\alpha=1.1,1.2,1.3$ (left to right) on uniformly refined covers with ten enrichment functions employed on every patch $\omega_{i}$ with a lumped overlap matrix. The dashed line indicates the accuracy of the employed reference solution.

order $O\left(10^{11}\right)$ whereas our flat-top PUM with stability transform and lumping using a comparable setup provides an error of $5 \cdot 10^{-3}$ Ha with 810 DOFs and an optimal condition number of 1 for the lumped overlap matrix.

Lastly, we examine the influence of the flat-top region on the eigenvalue convergence with the lumped overlap matrix. To this end, we consider various values of the stretch factor (14) $\alpha=1.1,1.2,1.3$. Figure 10 shows the convergence of the sum of the ten lowest eigenvalues for different values of $\alpha$. For these tests, we enrich each of the ten lowest states on every patch of our cover and use the stability transform (17). From the depicted plots it becomes clear that lumping provides better results for smaller stretch factors $\alpha$. The absolute value of the error grows with increasing $\alpha$ as well as the pre-asymptotic range of the convergence behavior. For fine enough covers, i.e., in the asymptotic range, the convergence behavior is very much in agreement with the consistent scheme. These observations can be explained by the structure of the error introduced by the lumping scheme [18]. Recall that our PUM degenerates to a DG approach with $\alpha=1$ and that the lumped mass matrix converges to the consistent matrix for $\alpha \rightarrow 1$. Thus, it is expected that the approximation quality of the computed eigenvalues will improve with smaller $\alpha$. The a priori determination of an optimal stretch factor $\alpha$ for a particular choice of the cover and employed local approximation spaces $V_{i}$, however, is the subject of current research.

\subsection{Gaussian potential}

As our second benchmark problem, we consider a periodic Gaussian potential [2]. This external potential is defined via

$$
V(\boldsymbol{x})=\sum_{\boldsymbol{R}} V_{g}(|\boldsymbol{x}-\boldsymbol{\tau}-\boldsymbol{R}|)
$$

with

$$
V_{g}(r)=-10 \exp \left(-\frac{r^{2}}{2.25}\right)
$$

where we sum over the lattice translation vectors $\boldsymbol{R}=i_{1} \boldsymbol{a}_{1}+i_{2} \boldsymbol{a}_{2}+i_{3} \boldsymbol{a}_{3}, i_{d}=-2, \ldots, 2(d=1,2,3)$. The unit cell $\Omega$ and the potential center $\tau$ are defined as in Section 4.1. For this experiment, 
we solve (4) subject to Bloch-periodic boundary conditions and choose $\boldsymbol{k}=(0.12,0.23,0.34)$ in reciprocal lattice coordinates. We choose the same approximation spaces and stretch factor $\alpha=1.1$ as in Section 4.1. The problem-dependent radial part $R_{n l}$ of the infinite box eigenfunctions is multiplied by $h(r, 10)$, i.e., $r_{0}=10$ au in (23). Also, as before, we are measuring the absolute error with respect to a cubic finite element reference solution computed on a $64 \times 64 \times 64$ mesh (with $\lambda_{1}^{\text {ref }}=-5.9605494576 \mathrm{Ha}$ and $\sum_{i=1}^{10} \lambda_{i}^{\text {ref }}=-26.309704717 \mathrm{Ha}$ ). For this example, we only show the convergence results using a lumped overlap matrix and increasing the enrichment radius on a fixed cover (analogous to the situation studied in Figure 99) to validate the method for Bloch-periodic boundary conditions. The results obtained for the Gaussian potential are shown in Figure 11. As for the harmonic oscillator example in Section 4.1, we see the expected convergence behavior of the non-enriched methods and a significant improvement of accuracy when increasing the number of patches that are enriched. Again, the benefits of enrichment are striking: for this particular example, we only need as few as 135 DOFs (with linear polynomials on a $3 \times 3 \times 3$ cover) to achieve the desired accuracy of $10^{-3} \mathrm{Ha}$ for the lowest state.

\section{Concluding remarks}

In this paper, we addressed two key issues in quantum mechanical calculations using enriched partition of unity finite element methods: the need to solve a generalized rather than standard eigenvalue problem, and the ill-conditioning of the associated system matrices. To address these, we developed a stable and efficient orbital-enriched flat-top partition of unity method to solve the required Schrödinger equation subject to Bloch-periodic boundary conditions in a general parallelepiped domain. To this end, we employed a stable transformation and variational mass lumping in a flat-top partition of unity formulation. Compared to PUFEM approaches used previously, the present method yields well- (or even optimally-) conditioned system matrices and a standard eigenvalue problem rather than a generalized one, while maintaining accuracy and systematic convergence to benchmark results.

With the above key issues resolved, future work will focus on the incorporation of nonlocal pseudopotentials, as occur in practical calculations involving all but the lightest elements, systematic determination of maximal $\alpha$ consistent with chemical accuracy with variational mass lumping, and efficient numerical cubature. A corresponding implementation for the Poisson equation will then enable full Kohn-Sham calculations.

\section{Acknowledgments}

This work was performed, in part, under the auspices of the U.S. Department of Energy by Lawrence Livermore National Laboratory under Contract DE-AC52-07NA27344. 

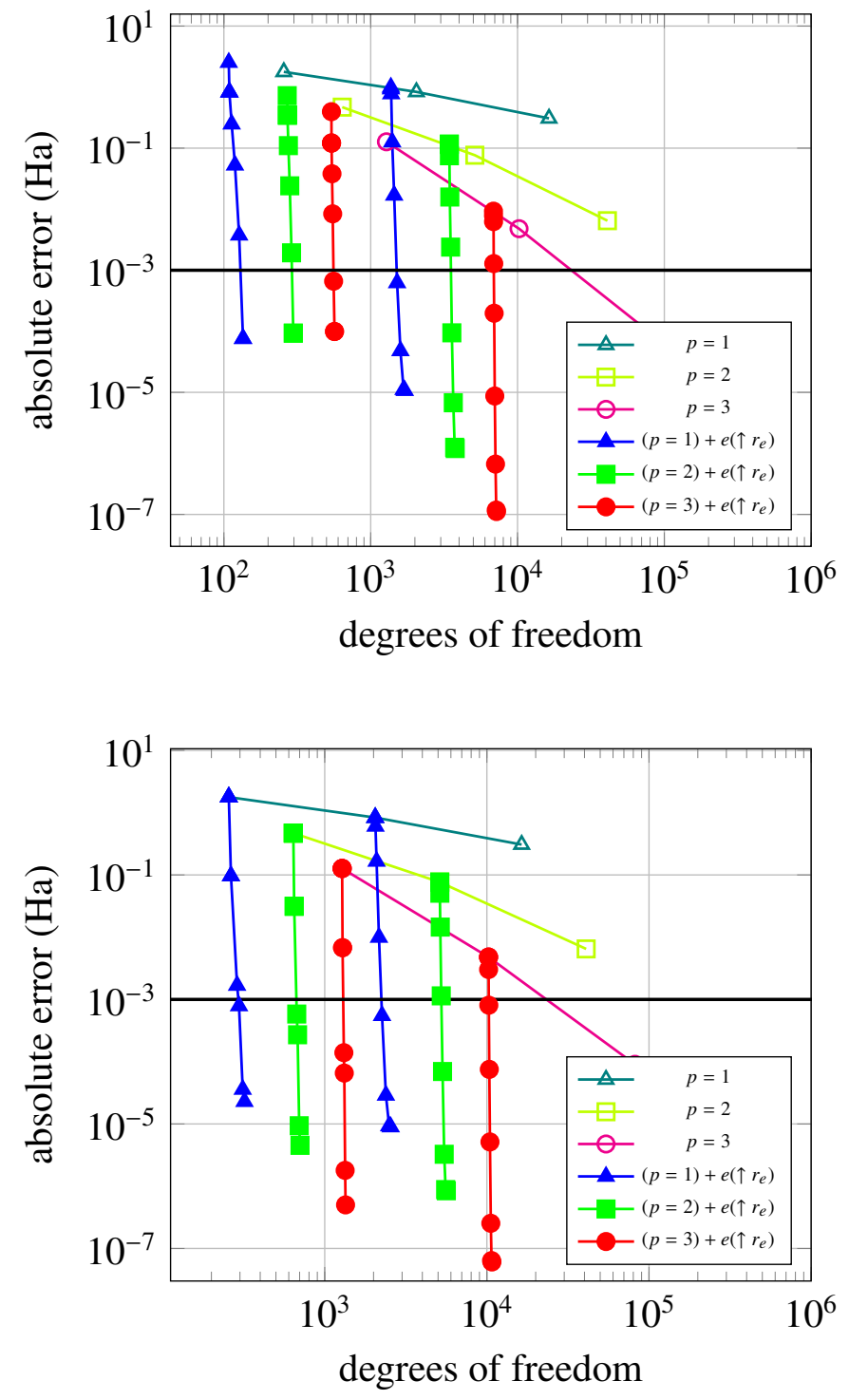

Figure 11: Convergence history of the lowest eigenvalue $\lambda_{1}$ for the periodic Gaussian potential (24) using different refinement schemes. We consider a purely polynomial approximation $(p=1,2,3)$ on a sequence of uniformly refined covers, and a refinement by increasing the enrichment radius (compare Figure 6) with a single enrichment function on a fixed uniform cover (top: $3 \times 3 \times 3,7 \times 7 \times 7$; bottom: $4 \times 4 \times 4,8 \times 8 \times 8$ ) that is labeled by $p=1,2,3+e \uparrow r_{e}$. All results were obtained with the lumped overlap matrix. 


\section{References}

[1] N. W. Ashcroft, N. D. Mermin, Solid State Physics, Holt, Rinehart and Winston, New York, 1976.

[2] N. Sukumar, J. E. Pask, Classical and enriched finite element formulations for Bloch-periodic boundary conditions, International Journal for Numerical Methods in Engineering 77 (8) (2009) 1121-1138.

[3] L. Lin, J. Lu, L. Ying, W. E, Adaptive local basis set for Kohn-Sham density functional theory in a discontinuous Galerkin framework I: Total energy calculation, Journal of Computational Physics 231 (4) (2012) 2140-2154.

[4] G. Zhang, L. Lin, W. Hu, C. Yang, J. E. Pask, Adaptive local basis set for Kohn-Sham density functional theory in a discontinuous Galerkin framework II: Force, vibration, and molecular dynamics calculations, Journal of Computational Physics 335 (2017) 426-443.

[5] J. E. Pask, N. Sukumar, Partition of unity finite element method for quantum mechanical materials calculations, Extreme Mechanics Letters 11 (2017) 8-17.

[6] S. Yamakawa, S. Hyodo, Electronic state calculation of hydrogen in metal clusters based on Gaussian-fem mixed basis function, Journal of Alloys and Compounds 356-357 (2003) 231-235.

[7] S. Yamakawa, S. Hyodo, Gaussian finite-element mixed-basis method for electronic structure calculations, Phys. Rev. B 71 (3) (2005) 035113.

[8] J. S. Chen, W. Hu, M. Puso, Orbital $h p$-cloud for solving Schrödinger equation in quantum mechanics, Comput. Methods Appl. Mech. Engrg. 196 (2007) 3693-3705.

[9] J. E. Pask, N. Sukumar, M. Guney, W. Hu, Partition-of-unity finite-element method for large scale quantum molecular dynamics on massively parallel computational platforms, Tech. Rep. LLNL-TR-470692, Department of Energy LDRD Grant 08-ERD-052 (March 2011).

[10] J. E. Pask, N. Sukumar, S. E. Mousavi, Linear scaling solution of the all-electron Coulomb problem in solids, International Journal for Multiscale Computational Engineering 10 (1) (2012) 83-99.

[11] A. S. Banerjee, L. Lin, W. Hu., C. Yang, J. E. Pask, Chebyshev polynomial filtered subspace iteration in the discontinuous Galerkin method for large-scale electronic structure calculations, Journal of Chemical Physics 145 (15) (2016) 154101.

[12] B. Kanungo, V. Gavini, Large-scale all-electron density functional theory calculations using an enriched finite-element basis, Phys. Rev. B 95 (2017) 035112.

[13] D. Davydov, T. Gerasimov, J.-P. Pelteret, P. Steinmann, Convergence study of the $h$-adaptive pum and the $h p$-adaptive fem applied to eigenvalue problems in quantum mechanics, Advanced Modeling and Simulation in Engineering Sciences 4 (1) (2017) 7. 
[14] I. Babuška, J. M. Melenk, The partition of unity finite element method: Basic theory and applications, Comput. Meth. Appl. Mech. Engrg. 139 (1996) 289-314.

[15] I. Babuška, J. M. Melenk, The partition of unity method, Int. J. Numer. Meth. Engrg. 40 (1997) 727-758.

[16] M. Griebel, M. A. Schweitzer, A particle-partition of unity method-Part II: Efficient cover construction and reliable integration, SIAM J. Sci. Comput. 23 (5) (2002) 1655-1682.

[17] M. A. Schweitzer, Stable enrichment and local preconditioning in the particle-partition of unity method, Numer. Math. 118 (1) (2011) 137-170.

[18] M. A. Schweitzer, Variational mass lumping in the partition of unity method, SIAM Journal on Scientific Computing 35 (2) (2013) A1073-A1097.

[19] F. Ihlenburg, Finite Element Analysis of Acoustic Scattering, Vol. 132 of Applied Mathematical Sciences, Springer-Verlag, New York, NY, 1998.

[20] P. A. Deymier (Ed.), Acoustic Metamaterials and Phononic Crystals, Spring Series in SolidState Sciences, Springer, New York, NY, 2013.

[21] J. D. Joannopoulos, S. G. Johnson, J. N. Winn, R. D. Meade, Photonic Crystals: Molding the Flow of Light, 2nd Edition, Princeton University Press, Princeton, NJ, 2008.

[22] M. A. Schweitzer, A Parallel Multilevel Partition of Unity Method for Elliptic Partial Differential Equations, Vol. 29 of Lecture Notes in Computational Science and Engineering, Springer, 2003.

[23] C. Klaar, A partition of unity method for quantum mechanical material calculations, Master's thesis, Rheinische Friedrich-Wilhelms-Universitt Bonn, Institut fr Numerische Simulation (2016).

[24] T.-P. Fries, T. Belytschko, The extended/generalized finite element method: An overview of the method and its applications, Int. J. Numer. Meth. Engrg. 84 (3) (2010) 253-304.

[25] M. A. Schweitzer, Generalizations of the finite element method, Cent. Eur. J. Math. 10 (1) (2012) 3-24.

[26] I. Babuška, G. Caloz, J. E. Osborn, Special finite element methods for a class of second order elliptic problems with rough coefficients, SIAM J. Numer. Anal. 31 (1994) 945-981.

[27] M. A. Schweitzer, Generalized finite element and meshfree methods, Habilitation, Univeristät Bonn (2008).

[28] M. Griebel, M. A. Schweitzer, A particle-partition of unity method-Part VII: Adaptivity, in: M. Griebel, M. A. Schweitzer (Eds.), Meshfree Methods for Partial Differential Equations III, Vol. 57 of Lecture Notes in Computational Science and Engineering, Springer, 2006, pp. 121-148. 
[29] M. A. Schweitzer, An adaptive hp-version of the multilevel particle-partition of unity method, Comput. Meth. Appl. Mech. Engrg. 198 (2009) 1260-1272.

[30] T. Strouboulis, I. Babuška, R. Hidajat, The generalized finite element method for Helmholtz equation: Theory, computation, and open problems, Comput. Meth. Appl. Mech. Engrg. 195 (37-40) (2006) 4711-4731.

[31] T. Strouboulis, R. Hidajat, I. Babuška, The generalized finite element method for Helmholtz equation. Part II: Effect of choice of handbook functions, error due to absorbing boundary conditions and its assessment, Comput. Meth. Appl. Mech. Engrg. 197 (5) (2008) 364-380.

[32] C. A. M. Duarte, J. T. Oden, $h p$ clouds - A meshless method to solve boundary value problems, Numer. Meth. for PDE 12 (1996) 673-705.

[33] T. Belytschko, Y. Y. Lu, L. Gu, Crack propagation by element-free Galerkin methods, Engrg. Frac. Mech. 51 (1995) 295-315.

[34] M. A. Schweitzer, A parallel multilevel partition of unity method for elliptic partial differential equations, Dissertation, Institut für Angewandte Mathematik, Universität Bonn (2002).

[35] A. Huerta, T. Belytschko, T. Fernández-Méndez, T. Rabczuk, Meshfree Methods, Vol. 1 of Encyclopedia of Computational Mechanics, Wiley, 2004, Ch. 10, pp. 279-309.

[36] N. Moës, J. Dolbow, T. Belytschko, A finite element method for crack growth without remeshing, Int. J. Numer. Meth. Engrg. 46 (1999) 131-150.

[37] I. Babuška, U. Banerjee, Stable generalized finite element method (SGFEM), Computer Methods in Applied Mechanics and Engineering 201-204 (Supplement C) (2012) 91-111.

[38] J. Hu, Y.-Q. Huang, H. Shen, The lower approximation of eigenvalue by lumped mass finite element method, Journal of Computational Mathematics 22 (4) (2004) 545-556.

[39] G. Strang, G. J. Fix, An Analysis of the Finite Element Method, Prentice-Hall, 1973.

[40] P. Tong, T. H. H. Pian, L. L. Bucciarblli, Mode shapes and frequencies by finite element method using consistent and lumped masses, Computers \& Structures 1 (4) (1971) 623-638.

[41] T. J. R. Hughes, The Finite Element Method: Linear Static and Dynamic Finite Element Analysis, Dover Civil and Mechanical Engineering Series, Dover Publications, 2000.

[42] Y. Zhou, Y. S. M. L. Tiago, J. R. Chelikowsky, Self-consistent-field calculations using Chebyshev-filtered subspace iteration, J. Comput. Phys. 219 (1) (2006) 172-184. 